\title{
$\$$ Research Square

\section{Heart Rate Variability Enhances the Accuracy of Non-invasive Continuous Blood Pressure Estimation Under Blood Loss}

\section{Guang Zhang}

Institute of Medical Support, Academy of Military Science

\section{Zongge Wang}

School of Life Sciences, TianGong University https://orcid.org/0000-0002-9630-0387

\section{Feixiang Hou}

School of Life Sciences, TianGong University

Huiquan Wang ( $\sim$ huiquan85@126.com)

School of Life Sciences, TianGong University https://orcid.org/0000-0002-7896-303X

\section{Feng Chen}

Institute of Medical Support, Academy of Military Sciences

\section{Ming Yu}

Institue of Medical Support, Academy of Military Sciences

\section{Jinhai Wang}

School of Life Sciences, TianGong University

\section{Research}

Keywords: cuff-less blood pressure, pulse transit time, partial least squares, HRV

Posted Date: October 26th, 2020

DOl: https://doi.org/10.21203/rs.3.rs-95428/v1

License: (c) (i) This work is licensed under a Creative Commons Attribution 4.0 International License. Read Full License

Version of Record: A version of this preprint was published at Review of Scientific Instruments on October 1st, 2021. See the published version at https://doi.org/10.1063/5.0037661. 


\title{
Heart rate variability enhances the accuracy of non-invasive continuous blood pressure estimation under blood loss
}

\author{
Guang Zhang ${ }^{1}$, Zongge Wang ${ }^{1,2}$, Feixiang Hou ${ }^{1,2}$, Huiquan Wang ${ }^{2}$, , Feng Chen ${ }^{1}$ Ming $\mathrm{Yu}^{1}$, Jinhai Wang ${ }^{2}$
}

\begin{abstract}
Background: To propose a new method for real-time monitoring of human blood pressure under blood loss (BPBL), this article combines pulse transit time (PTT) and heart rate variability (HRV) as input parameters in order to establish a model for the estimation of BPBL.
\end{abstract}

Methods: Effective parameters such as PTT, R-R internal (RRI), and HRV were extracted and used to establish the blood pressure (BP) estimation. Three BP estimation models were established: the PTT model, the RRI model, and the HRV model, and they were divided into experimental group and control group. Finally, the effects of different estimation models on the accuracy of BPBL estimation were evaluated based on the experimental results.

Results: The Pearson correlation coefficients R were $0.7731,0.8943$ and 0.9169 for the PTT model, the RRI model, and the HRV model, respectively. The root means square error of the estimation set (RMSEP) were $16.83 \mathrm{mmHg}, 11.87 \mathrm{mmHg}$ and $10.59 \mathrm{mmHg}$, respectively.

Conclusion: The results suggest that the accuracy of the BPBL estimated by the RRI and HRV models is better than that of the PTT model, which means that both RRI and HRV can enhance the accuracy of BPBL estimation, and HRV seems to be more effective in improving the accuracy of BP prediction compared to RRI. These results provide a new idea for other scholars in the field of BPBL estimation research.

Key words: cuff-less blood pressure, pulse transit time, partial least squares, HRV

\section{Background}

Hemorrhagic shock constitutes the most common cause of death in patients who die at the scene of an accident or battlefield 
extremely serious consequences or even can be fatal [6-8]. The blood pressure (BP) of the human body changes rapidly during

blood loss which cannot be monitored dynamically [8], hence, it will greatly affect the timely judgment of the condition and

the effect of any treatment provided. Therefore, it is important to establish a non-invasive, fast, simple, and effective method of BP estimation to monitor its change under blood loss. However, on the global level, there is still a large gap in the related research in this field. and increase the BP through spontaneous regulatory actions. Therefore, the monitoring of cardiac autonomic nerve regulation

11 will help improve the accuracy of BP estimation. Heart rate variability (HRV) reflects several aspects of cardiac autonomic 12 nerve activity and is one of the most important indicators of cardiac autonomic nervous regulation [9-11]. Therefore, this 13 study combines HRV and the existing pulse transit time (PTT) theory to establish a novel BP estimation model, which is 14 able to provide continuous and efficient.

Compared with the traditional BP measurement method, the PTT method has attracted great attention in recent years because of its advantage of being a non-invasive, continuous, and cuff-less BP measuring method. Much research has been conducted on the method of estimating the BP based on PTT. Wong et al. [12] and Gesche et al. [13] have shown that the systolic BP (SBP) highly correlates with PTT, which also verifies the feasibility of predicting BP values based on PTT. Gesche et al. [13], Huynh et al. [14], and Mukkamala et al. [15] have provided the formula for calculating the BP value based on PTT and proved the reliability of the method. Ding et al. [16], Tao et al. [17] and Liu et al. [18] have adopted different methods to optimize the PTT method as well as improve the accuracy of predicting BP values. In addition to the theoretical research, Zheng et al. [19] applied the PTT method to continuously monitor BP at night and verified its feasibility for the continuous monitoring of BP. Others used the PTT method in personal health care to continuously measure human BP [20], they combined the PTT method with a chest sensor and performed a Mean arterial pressure (MAP) estimation on the chest through a corresponding calibration strategy [12], or used the PTT method to measure BP during and after dynamic exercise 
[21] which have all yielded in favorable results.

Although much research has been conducted which has yielded in a series of significant results in this field, there are still some certain limitations in current research outputs. Firstly, there is a large gap in the estimation and analysis of BPBL in terms of accuracy. Given that the scenario of blood loss is very common in the clinic, continuous monitoring and early warning of decreasing BP in the case of blood loss is important, which is what currently is lacking in the relevant BP research. In addition, there are still differences in the PTT-based BP estimation methods which are tied to individual patients, leading to a decrease in the accuracy of the results. Unfortunately, because the physiological states are not exactly the same and its effects cannot be completely eliminated in different individuals, their estimated BP will be different, hence, a standard continuous BP measurement method that is suitable for most people may be difficult to achieve.

In this article, R-wave to R-wave internal (RRI) and HRV will be used as input feature parameters to establish the BP estimation model. RRI and HRV are common indicators to measure the physiological status of different individuals, and, when used as input parameters to establish the BP estimation model, the errors caused by individual differences can effectively be reduced and the accuracy of BP estimation can be improved. This study uses RRI and HRV to establish different BP estimation models and evaluates each model based on experimental results, exploring the impact of different input parameters on the accuracy of BP estimation under blood loss. The results of this study may be helpful for other people who are conducting BP estimation research, and it is hoped to provide an additional contribution to the development in this field.

\section{Methods}

\section{Basic theory of blood loss}

When blood loss occurs, the blood volume in the blood vessels decreases, which in turn causes the BP to decrease. At the same time, the decrease in BP will be fed back to the nerve center, initiating a series of adjustment activities to increase BP, including accelerated blood pumping to increase cardiac output and blood vessel contraction. In order to better estimate the 

related to blood loss were determined in order to estimate BPBL. superimposes on the DC component [23]. The DC component is related to the blood volume in the blood vessel, and will slowly change

54 due to respiration and changes in the diameter of the blood vessel.

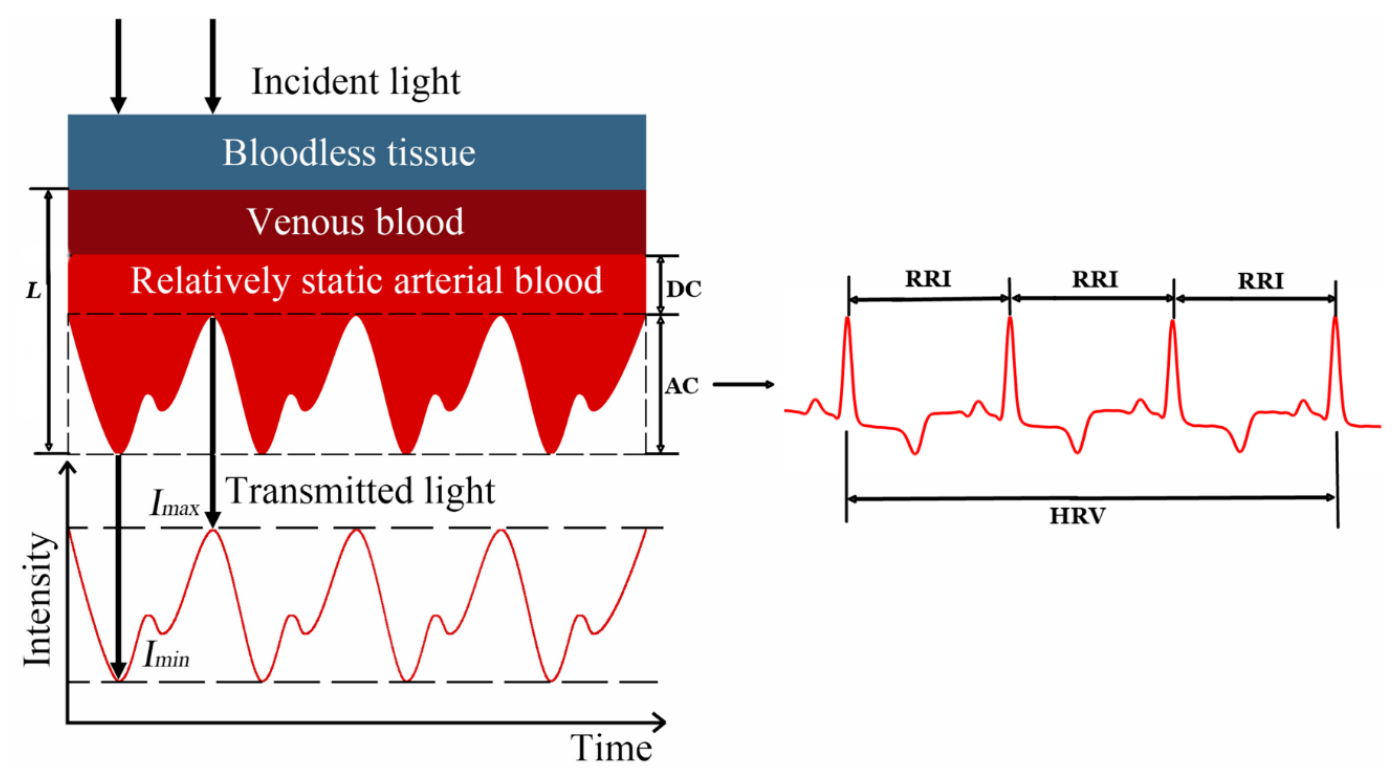

Fig. 1. Basic theory of blood loss.

Since there is a certain functional relationship between the total blood volume $\mathrm{V}$ of the venous blood $\left(V_{\text {ven }}\right)$, the arterial blood $\left(V_{\text {art }}\right)$ and the blood vessel diameter $\mathrm{L}$, it is assumed that the following formula exists between them:

$$
\begin{gathered}
V=V_{\text {ven }}+V_{\text {art }} \\
V_{\text {art }}=V(D C)+V(A C) \\
L=f(V)
\end{gathered}
$$

When blood loss occurs, the blood volume in the blood vessel decreases, and according to formula (3), the blood vessel diameter L 
will also decrease. Due to the change in diameter L, the static arterial blood (DC) component of the PPG waveform will also change.

64 At the same time, the heart will speed up when blood is lost, trying to maintain enough blood supply to the organs, which will change the heart rate. Since $\mathrm{AC}$ is determined by heart rate, it will also change significantly during the blood loss process. For the estimation of BPBL, the AC and DC parameters that are closely related to blood loss are of significance. Therefore, in this study the two parameters AC and DC were extracted for the construction of the BPBL model. In addition, since the human body performs autonomous neuromodulation to suppress BP reduction during blood loss, the quantification of the neuromodulation process will help improve the accuracy of the BPBL estimation. One method of assessing autonomic modulation is through HRV, which is usually calculated by RRI and is a common method for monitoring autonomic nerve activity [10-11]. In this study, the time domain parameters (SDNN and HRV signals were also extracted and utilized to estimate the BPBL.

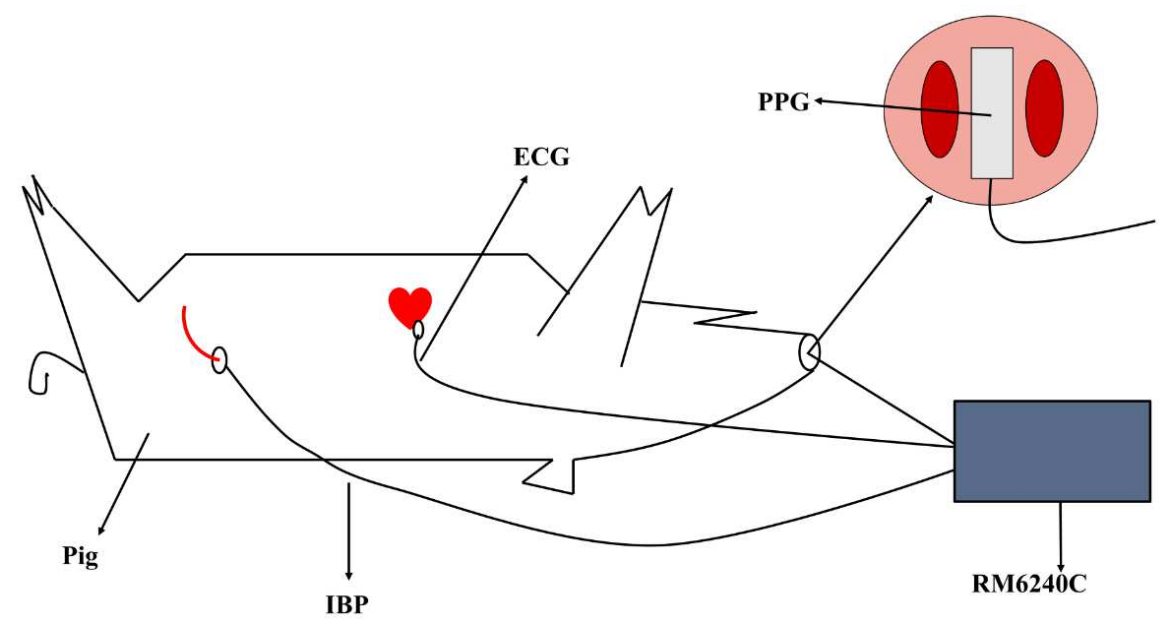

Fig. 2. Experimental scene diagram.

\section{Experimental process}

In this study, Landrace pigs were used as experimental objects. They were fixed on the operating table and anesthetized during the experiment. As shown in Fig. 2, three signals were detected during the experiment: the first signal was the ECGs signal detected around the heart, which was obtained via the unipolar lead method. The second signal was the pulse signal located at the nose, which was obtained via the PPG method. The third signal was the invasive continuous blood pressure 
(IBP) located in the left femoral artery of the pig. IBP is regarded as the gold standard for BP measurement and can be used

81 for modeling calculations and verification. All three signals were collected from the pigs simultaneously and were displayed

82 by the Chengdu Instrument RM6240C multi-channel physiological signal acquisition system, with experimental data being

83 recorded throughout the process, and all signals being sampled at $1000 \mathrm{~Hz}$.

84 The experiments were conducted on a total of five pigs with a weight range of $31 \pm 8.5 \mathrm{~kg}$. The specific experimental

process is shown in Fig. 3. At the beginning of the experiment, it was crucial to record the baseline physiological data of the experiment.

\section{Baseline 10 min}

pigs in a stable state. It was made sure the pigs were under anesthesia, and $200 \mathrm{ml}$ of blood was withdrawn via the pigs' carotid artery. The corresponding time points were recorded and the physiological changes of the pigs were observed. When the $\mathrm{BP}$ and $\mathrm{HR}$ of the pigs reached a relatively stable state, the pigs were bled again $(200 \mathrm{ml}-400 \mathrm{ml})$ and the corresponding data were recorded. As soon as the pigs lost blood at a certain level, they were given another blood transfusion. The above process was repeated several times, with the number of repetitions determined according to the specific experimental situations. Because the blood volume of the pigs is in linear relationship with their body weight, the number of the bloodletting instances was determined according to the weight of the pigs and the specific experimental conditions, in order to achieve the aim of this study. For this study, a total of 10 sections of valid blood loss data were extracted during the experiment.

(1)

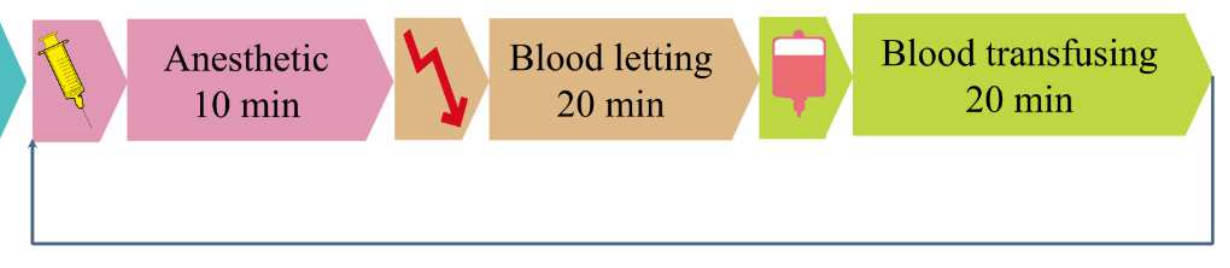

Fig. 3. Introduction to the experimental process.

\section{Feature parameter extraction}

After the experiment, the main characteristic parameters such as PTT, RRI, and HRV were extracted from the ECG and PPG signals collected from the experiments. As shown in Fig. 4 (a), The PTT refers to the time that the pulse wave travels from the 
heart to the surrounding branch blood vessels during the pumping of the heart. In this study, PTT was regarded the time of

the pulse wave traveling from the heart to the pig's nose.

corresponding algorithm was located. Due to the ECG and PPG signals being collected simultaneously, and the pulse wave

signal being transmitted from the heart to the pig's nose, the ECG signal preceded the PPG signal for a period of time, and

there was only one PPG peak between two adjacent R peaks. In the same way, all the data segments that met these criteria were extracted, and the remaining data points were regarded as outliers and discarded. After all the valid data segments were obtained, the R peak point of the ECG signal was used as the starting point, and the peak point of the adjacent PPG signal data were extracted to establish the BPBL estimation model.

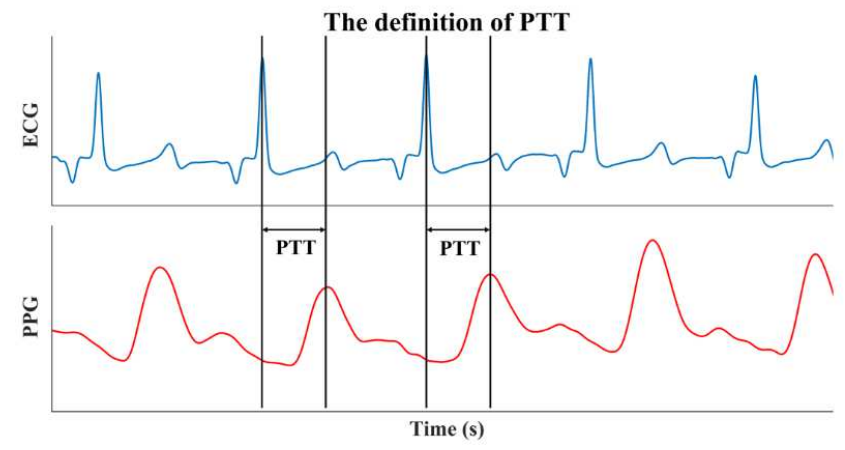

(a)

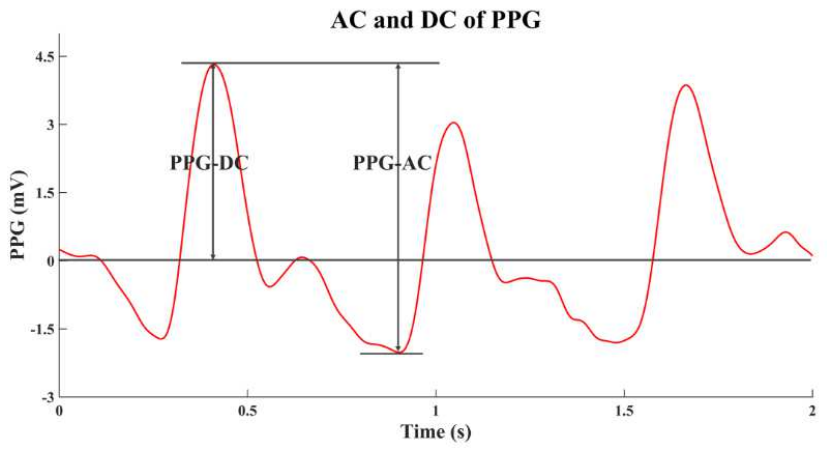

(b)

Fig.4. The extraction of PTT, AC, and DC.

In addition to the PTT parameters, other characteristic parameters were also extracted to establish the BPBL estimation model. As shown in Fig. 5, these parameters include two parts: the first component is the pulse amplitude (AC) and peak (DC) extracted from the PPG signal. AC/DC as the input parameter was also calculated to reduce the interference of individual differences. The second component is the RRI and HRV (SDNN, RMSSD, and HF) extracted from the PPG signal, which is the key parameter of this experiment. Combined with PTT and PTT/ RRI, a total of nine characteristic parameters were extracted in the study which were mainly used as input parameter to establish the BPBL estimation model. 


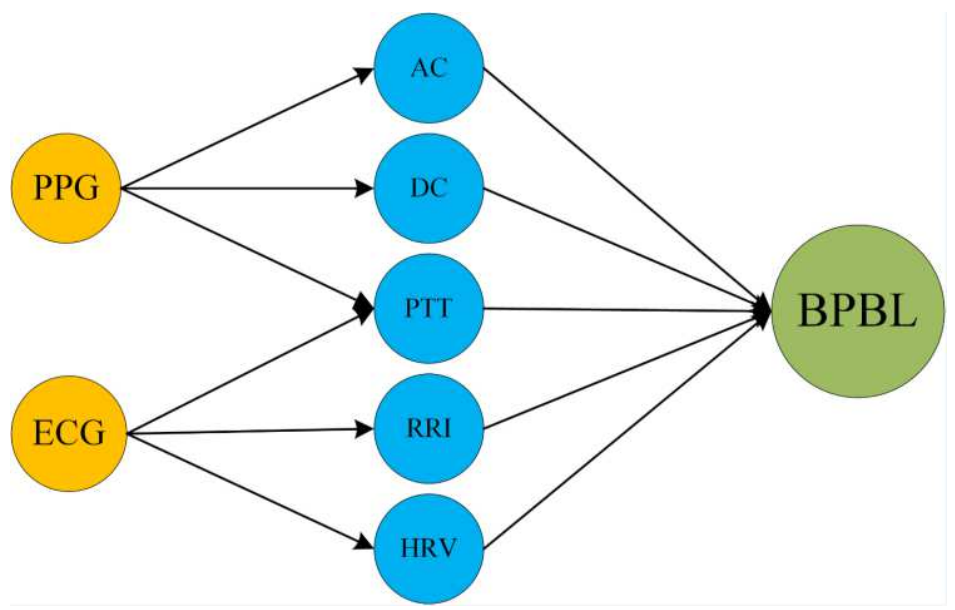

Fig. 5. Parameters for estimating BPBL

\section{Construction of the BPBL estimation model}

Because the BP estimation model constructed in this study has multiple input parameters which show a strong correlation

between each other, the partial least squares (PLS) method was deemed suitable to build a BP estimation model under blood

loss. In this study, three BP estimation models were constructed: the PTT model, the RRI model, and the HRV model. The

PTT model refers to a commonly used BP estimation model based on PTT, and the input characteristic parameters included

PTT, AC, DC, AC/DC. The RRI model and the HRV model refer to the model constructed by adding the RRI and the HRV,

respectively, based on the input parameters of the PTT model. In the described experimental procedure of this study, the

PTT model was set as the control group, while the RRI model and the HRV model were set as the experimental group. The input characteristic parameters of the different models are shown in Table 1.

Table 1. Comparison of Input Parameters of Different Models

\begin{tabular}{cc}
\hline \hline BPBL Model & Input Parameters \\
\hline PTT model & PTT, AC, DC, AC/DC \\
RRI model & PTT, AC, DC, AC/DC, RRI, PTT/RRI \\
HRV model & PTT, AC, DC, AC/DC, SDNN, RMSSD, HF \\
\hline \hline
\end{tabular}


the training set and the estimation set. The training set and the estimation set accounted for $9 / 10$ and $1 / 10$ of the total sample,

134 respectively. After every nine data sets in the total sample, one data set was selected to form the estimation set, and the 135 remaining data was allocated to the training set. In this study, PLS was used to establish a model between 1107 samples and 136 the SBP values in the training set. Then the established training model was used to estimate the SBP values of 122 samples 137 in the estimation set.

138 The correlation between the estimated value and the actual value was established by evaluating the predictive ability of the model through the following indicators: the root means square error (RMSEC) and the correlation coefficient of the training set (Rt), and the root mean square error (RMSEP) and the correlation coefficient of the estimation set (Re).

141 When the correlation coefficient is larger and the root mean square error is smaller, it indicates that the model has a better 142 predictive ability.

\section{Results}

144 In the experiment, three different BPBL estimation models were constructed with the input feature parameters mentioned 145 previously based on the partial least squares (PLS). Three different BPBL estimation models were tested with the same 146 experimental data, and the experimental results of the training set and the estimation set of SBP under blood loss were 147 obtained. There were 1229 sample points of all models. The P-values in table 2 shows that all experimental results were 148 statistically significant. The detailed experimental results of each model were shown in Table 2 and Fig. 6.

149 The correlation coefficient (Rp) and the root mean square error (RMSEP) were calculated in our study to assess the 150 accuracy of the estimated BPBL values. According to the Table 2, the Pearson correlation coefficient of the SBP and IBP 151 estimated by the PTT model of the experimental group, was lowest with $0.77(\mathrm{P}<0.01)$, while the Pearson correlation 152 coefficients estimated by the RRI model and the HRV model of the experimental group, reached $0.89(\mathrm{P}<0.01)$ and 0.92 $153(\mathrm{P}<0.01)$, respectively. In addition, the root mean square error of the test set in the PTT model was the largest with 16.83 , 154 while the mean square error of the estimation set in the RRI model and the HRV model was 11.87 and 10.59 respectively. 
Table 2. Comparison of experimental results of three models.

\begin{tabular}{ccccccc}
\hline \hline Blood Loss Model & $\begin{array}{c}\text { Optimal } \\
\text { Factor }\end{array}$ & Rt & RMSEC & Rp & RMSEP & P-value \\
\hline PTT model & 3 & 0.73 & $18.1 \mathrm{mmHg}$ & 0.77 & $16.8 \mathrm{mmHg}$ & $* * *$ \\
RRI model & 5 & 0.89 & $12.2 \mathrm{mmHg}$ & 0.89 & $11.9 \mathrm{mmHg}$ & $* * *$ \\
HRV model & 6 & 0.91 & $11.1 \mathrm{mmHg}$ & 0.92 & $10.6 \mathrm{mmHg}$ & $* * *$ \\
\hline \hline
\end{tabular}

*** represents $\mathrm{P}<0.01$. Rt and $\mathrm{Rp}$ represent the Pearson correlation coefficients of the training set and prediction set,

respectively. RMSEC and RMSEP represent the root mean square error of the training set and prediction set, respectively.
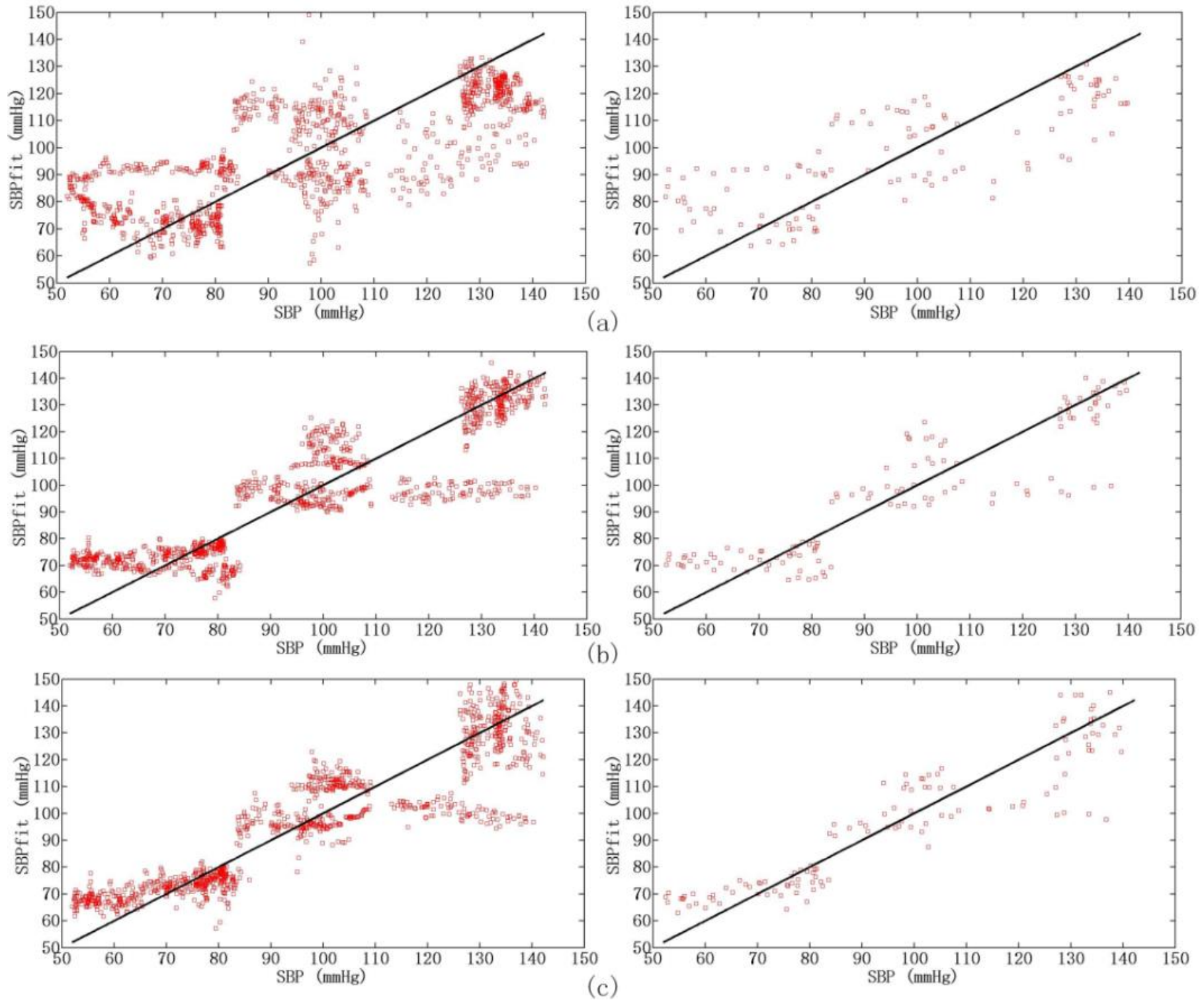

158

159

160

161

Fig. 6. The results of the training set and prediction set of three blood loss models

Therefore, it is obvious that the correlation estimated by the RRI model and the HRV model is better than that of the PPT model. Moreover, the estimated effect of the BP value of the HRV model is greater than that of the RRI model. 


\section{Discussion}

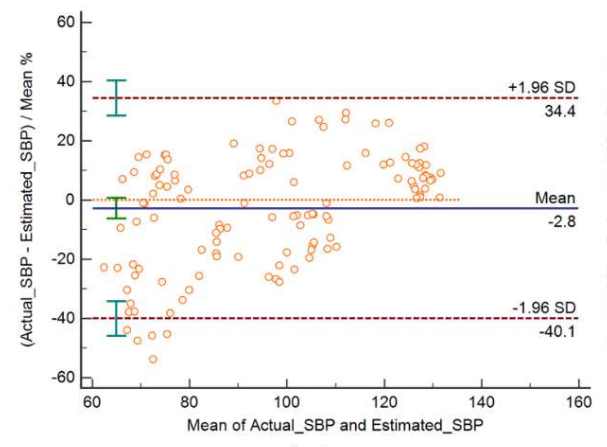

(a)

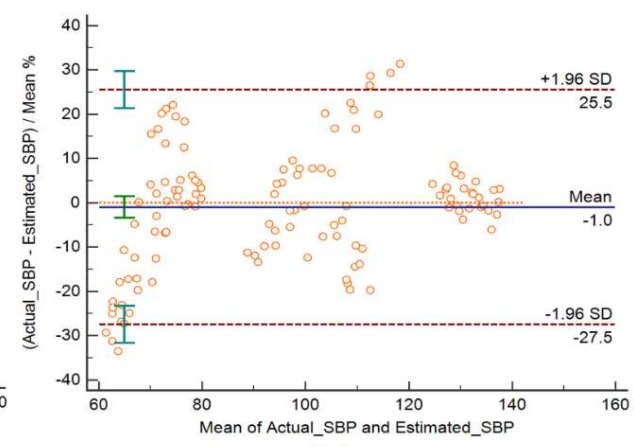

(b)

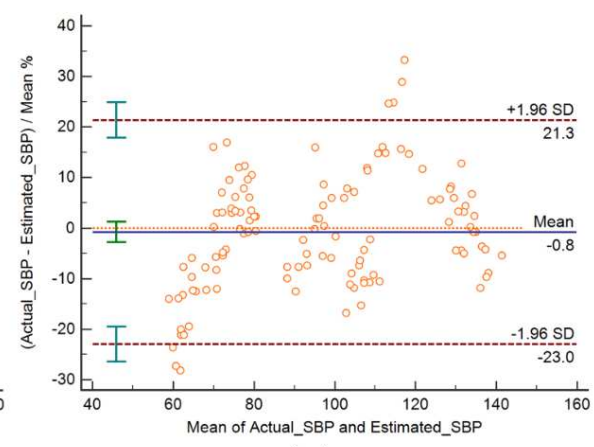

(c)

Fig. 7. Bland-Altman plot of the difference between actual SBP and estimated SBP in three models.

The experimental group has a better effect on BP estimation

174 Compared with the control group, the correlations of the RRI model and the HRV model increased by $10.1 \%$ and $18.6 \%$, respectively. At the same time, compared with the PTT model, the mean square error was reduced by $29.5 \%$ and $37.1 \%$, respectively. Therefore, it is obvious that the experimental group achieve a much better accuracy than the control group. 
when HRV and R-R intervals are added as input parameters. It could be speculated that this is due to HRV and R-R intervals

being more relevant to the characteristics of the human body, and when used as input parameters to establish the estimation

model they may reduce the impact of individual differences and increase the accuracy of BP estimation.

HRV Enhances the Accuracy of BPBL Estimation significantly enhance the estimation accuracy of BPBL.

\section{HRV is more meaningful than RRI}

By comparing the estimation results of the RRI model and the HRV model of the experimental group, the Pearson correlation coefficient of SBP and IBP estimated by the HRV model is higher than that of the RRI model, and the mean square error of the estimated value of the HRV model is lower than that of the RRI model. This shows that HRV has a better correlation with BPBL, and can greatly enhance the accuracy of BPBL estimation.

The HRV indicators used in this study are calculated by RRI, since RRI may contain more information than HRV, such as heart rate. However, the comparison of the two models in the experimental group indicated that HRV is better than RRI in estimating the BPBL. Therefore, it can be argued that when blood loss in pigs occur, they mainly regulate it through the 
provides a more effective extraction of blood loss information and largely removes redundant information, which makes

HRV a more desirable parameter to BPBL estimation.

\section{Limitations}

There are several limitations in this study. The amounts of data used is not sufficient, more blood loss data are needed to

increase the reliability of the BPBL estimation model. Future research should obtain more data to generate a more accurate

BPBL model. Furthermore, a lot of parameters were extracted for this experiment, but a few of these parameters may only give a small contribution to the overall results, and more relevant parameters have not been identified. The relevance of other parameters to the BPBL needs to be verified, and more effective input parameters are to be used to improve the BPBL model.

\section{Conclusions}

211 In this article, we established three BPBL estimation models and compared their estimation results. The results of this study 212 and the BPBL estimation model provide evidence that the RRI and HRV are good correlators with the BPBL and enhance 213 the accuracy of the BPBL estimation. The comparison of the three models suggest that the HRV model, based on the 214 characteristic parameters of HRV and PTT, has a better estimation of results during blood loss, thereby improving the 215 accuracy of the BPBL estimation and therefore, being more suitable as a blood loss estimation model. This research on the 216 process of blood loss and BP can be expanded to other fields, which will become one of its future directions. And it is hoped 217 that this article will serve as a guidance for related research attempts in the future.

218 Abbreviations

219 BP: blood pressure; HRV: heart rate variability; PTT: pulse transit time; BPBL: blood pressure under blood loss; SBP: 220 systolic blood pressure; MAP: mean arterial pressure; IBP: invasive blood pressure; RRI: R-R internal; PPG: 221 photoplethysmography; PLS: partial least squares; Rt: correlation coefficient of the training set; Re: correlation coefficient 222 of the estimation set; RMSEP: root means square error of the estimation set.

\section{Declarations}




\section{Ethics approval and consent to participate}

225 This study strictly followed the animal experiment guidelines and was approved by the Medical Ethics Committee of the

226 Chinese People's Liberation Army General Hospital (S2020-045-01).

227 Consent for publication

228 Not applicable.

229 Availability of data and materials

230 Due to the need for confidentiality, the datasets analyzed during the current study are not publicly available but are available

231 from the corresponding author on reasonable request.

232 Competing interests

233 The authors declare that they have no competing interests.

\section{Funding}

235 The work was supported by National Key R\&D Program of China (Grant Number: 2017YFC0806402, 2017YFC0806404,

236 2017YFC0806406) and Science and Technology Program of Tianjin, China (Grant Number: 18ZXJMTG00060), and Tianjin

237 Science and Technology Plan Project (Grant No. 18ZXRHSY00200).

238 Authors' contributions

239 GZ and HQW conceived and designed this study. ZGW and FXH wrote this article. JHW helps to check and revise articles. FC

240 and MY contributed to material support. All authors read and approved the final manuscript.

241 Acknowledgements

242 Not applicable.

243 Authors' information (optional)

$244 *$ *Correspondence: huiquan85@ 126.com

245 1Institute of Medical Support, Academy of Military Sciences, Tianjin 300161, China.

246 2School of Life Sciences, TianGong University, Tianjin 300387, China.

247 Full list of author information is available at the end of the article. 


\section{References}

1. Marius N, Wilhelm B, Martin S, René S. A Systematic Review of Neuroprotective Strategies during Hypovolemia and Hemorrhagic Shock. International Journal of Molecular Sciences, 2017; 18(11): 2247.

2. Noa K, Eitan H, Nairouz F, Nadav I, et al. Endovascular Electrical Stimulation - A Novel Hemorrhage Control Technique. IEEE Trans. Biomed. Eng. 2019; 66(7):2072-2080.

3. Major UK, Brig GN, Corporal AL, et al. Analysis of the causes of death of casualties in field military setting. Mil. Med. 2012; 177(9): 1065-1068.

4. Butler FK, Kotwal RS, Buckenmaier CC, et al. A Triple-Option Analgesia Plan for Tactical Combat Casualty Care: TCCC Guidelines Change 13-04. J Spec. Oper. Med. 2014; 14(1): 13-25.

5. Guillaume T, Anatole H, Sebastien T, Hiromi K, et al. Microcirculatory alterations in traumatic hemorrhagic shock. Crit. Care Med. 2014; 42(6): 14331441.

6. Janet ML, Mark JM, Chen YF, Antonio B, et al. The systemic immune response to trauma: an overview of pathophysiology and treatment. Lancet, 2014; 384(9952): 1455-1465.

7. Jonathan JM, Richard EG, Jan OJ, Jeremy WC, et al. A systematic review of the use of resuscitative endovascular balloon occlusion of the aorta in the management of hemorrhagic shock. J Trauma Acute Care Surg. 2016; 80(2): 324-334.

8. Tobias M, Michael K, Michael P, et al. Attenuation of Multiple Organ Damage by Continuous Low-Dose Solvent-Free Infusions of Resveratrol after Severe Hemorrhagic Shock in Rats. Nutrients, 2017; 9(8):889.

9. Pecanha T, Forjaz CLD, Low DA. Passive Heating Attenuates Post-exercise Cardiac Autonomic Recovery in Healthy Young Males. Frontiers in Neuroscience, 2017;11(727).

10. Scott M, Graham KS, Oam GMD. Cardiac Autonomic Responses during Exercise and Post-exercise Recovery Using Heart Rate Variability and Systolic Time Intervals - A Review. Frontiers in Physiology, 2017; 8 (301).

$8(301)$.

11. Marianne PC, Jayme NJ, Bruna MC, Aline FBB, et al. Effects of functional training on geometric indices of heart rate variability. J Sport Health Sci. 2016; 5(2): 183-189.

12. Wong MYM, Poon CCY, Zhang YT, An evaluation of the cuffless blood pressure estimation based on pulse transit time technique: a half year study on normotensive subjects. Cardiovasc Eng. 2009; 9(1): 32-38.

13. Gesche H, Grosskurth D, Kuechler G, Patzak A. Continuous blood pressure measurement by using the pulse transit time: comparis on to a cuff-based method. European Journal of Applied Physiology, 2012; 112(1): 309-315.

14. Huynh TH, Jafari R, Chung WY. Noninvasive Cuffless Blood Pressure Estimation Using Pulse Transit Time and Impedance Plethysmography. IEEE Trans. Biomed. Eng. 2019; 66(4): 967-976.

15. Mukkamala R, Hahn JO, Inan OT, Mestha LK, et al. Toward Ubiquitous Blood Pressure Monitoring via Pulse Transit Time: Theory and Practice. IEEE Trans. Biomed. Eng. 2015; 62(8): 1879-1901.

16. Ding XR, Zhang YT, Liu J, Dai WX, et al. Continuous Cuffless Blood Pressure Estimation Using Pulse Transit Time and Photoplethysmogram Intensity Ratio. IEEE Trans. Biomed. Eng. 2016; 63(5): 964-972.

17. Tao W, Ji XY, Xu XD, Islam MA, et al. Antimonene Quantum Dots: Synthesis and Application as Near-Infrared Photothermal Agents for Effective Cancer Therapy. Angew. Chem Int Ed Engl. 2017; 56(39): 11896-11900.

18. Liu J, Yan BPY, Dai WX, Ding XR, et al. Multi-wavelength photoplethysmography method for skin arterial pulse extraction. Biomed Opt. Express, 2016; 7(10): 4313-4326.

19. Zheng YL , Yan BP, Zhang YT, Poon CCY, et al. An armband wearable device for overnight and cuff-less blood pressure estimation. IEEE Trans. Biomed. Eng. 2014; 61(7): 2179-2186.

20. Yoon Y, Cho JH, Yoon G. Non-constrained blood pressure monitoring using ECG and PPG for personal healthcare. J Med Syst, 2009; 33(4): 261-266.

21. Liu Q, Yan BP, Yu CM, Zhang YT, et al. Attenuation of systolic blood pressure and pulse transit time hysteresis during exercise and recovery in cardiovascular patients. IEEE Trans. Biomed. Eng. 2014; 61(2): 346-352.

22. Wang YY, Li G, Wang HQ, Zhou M, et al. Dynamic Spectrum for noninvasive blood component analysis and its advances. Applied Spectroscopy 


\section{Figure legend}

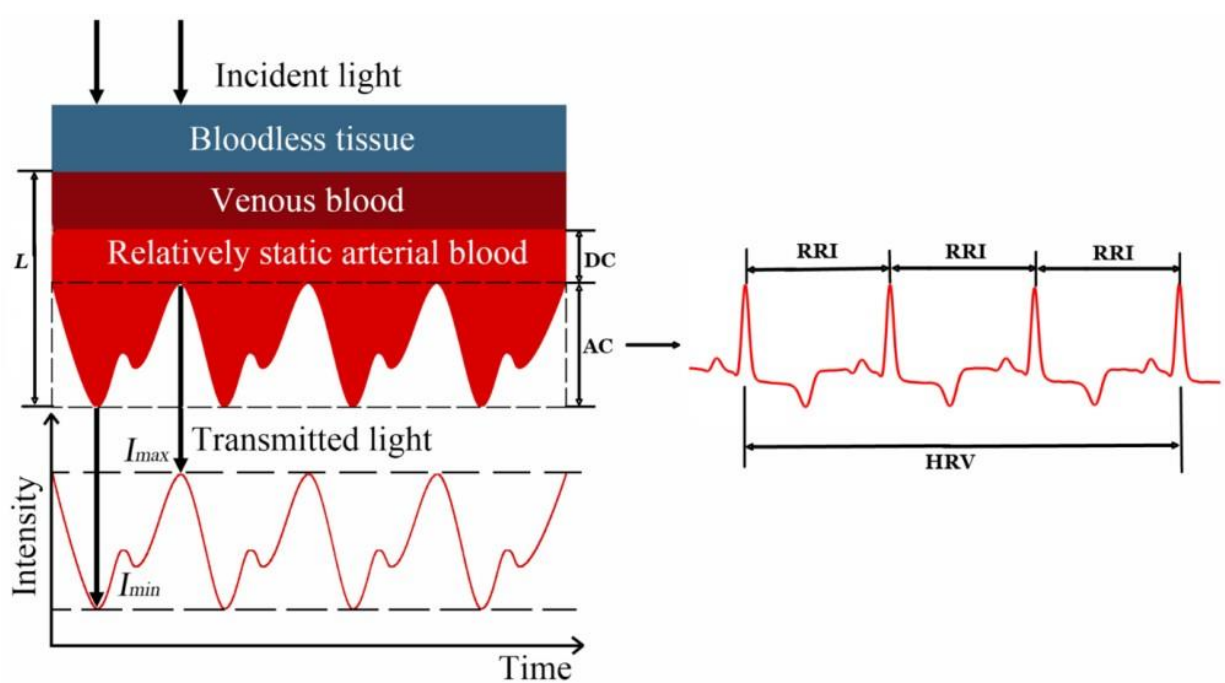

Fig. 1. Basic theory of blood loss. On the left side of the picture, the blood in the blood vessel is composed of dynamic components and static components. The near-infrared light emitted by PPG passes through the bloodless tissue layer and blood of the human body and reflects back and is received by the sensor. Since the light intensity change curve of the reflected light is closely related to the pulse, PPG technology can be used to obtain the pulse wave waveform, and the AC and DC can be extracted from the pulse wave. In addition, $\mathrm{AC}$ is depending on the heart rate, so we calculated RRI to reflect blood changes together.

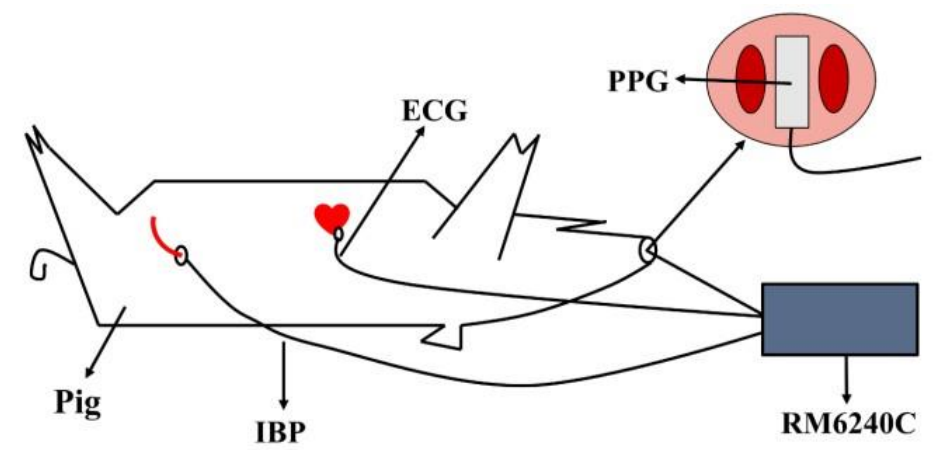

Fig. 2. Experimental scene diagram. The picture shows the side view of the pig on the operating table. During the experiment, we used the Chengdu Instrument RM6240C multi-physiological channel acquisition system to collect signals. We collected the PPG, ECG and IBP signals of the pig at the nose, chest and left femoral artery, respectively. 


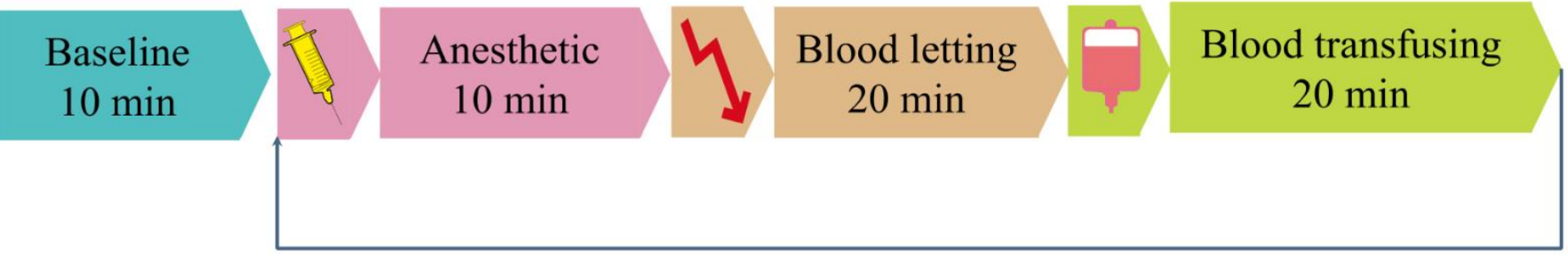

Fig. 3. Introduction to the experimental process. During the experiment, bloodletting and blood transfusion were performed when the text for further details.

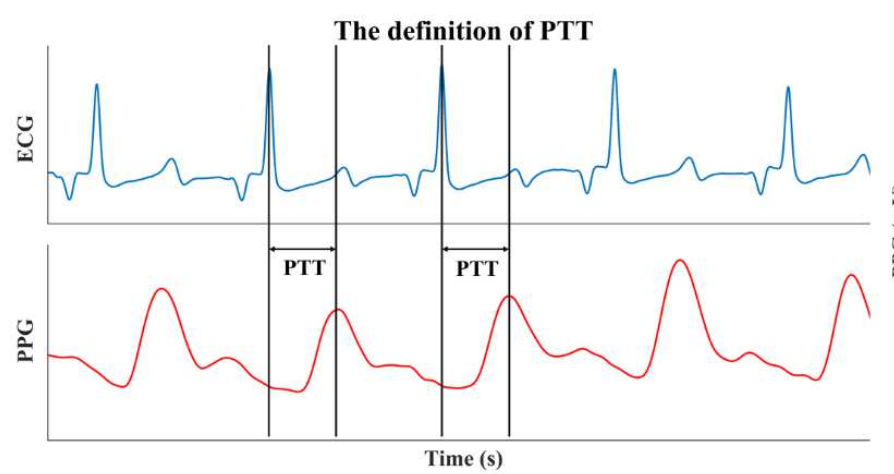

(a)

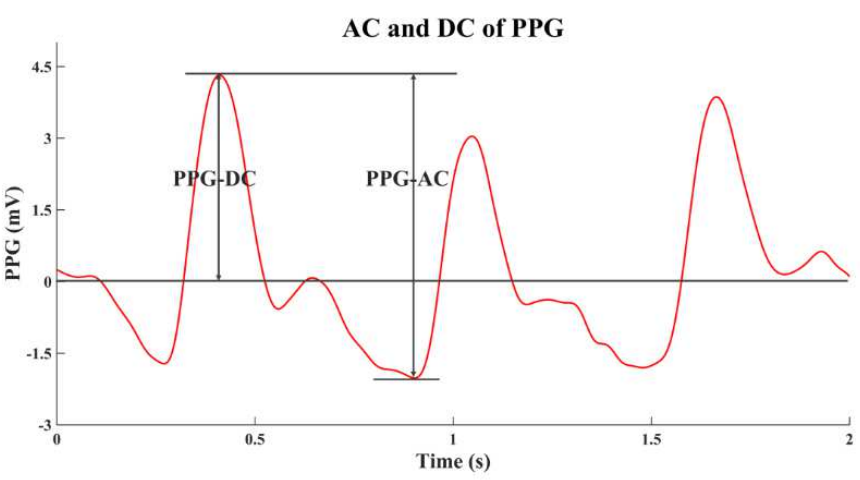

(b)

Fig.4. The extraction of PTT, AC, and DC. (a) The PTT refers to the time that the pulse wave travels from the heart to the surrounding branch blood vessels during the pumping of the heart. (b) DC and AC refer to the peak value and amplitude of PPG respectively.

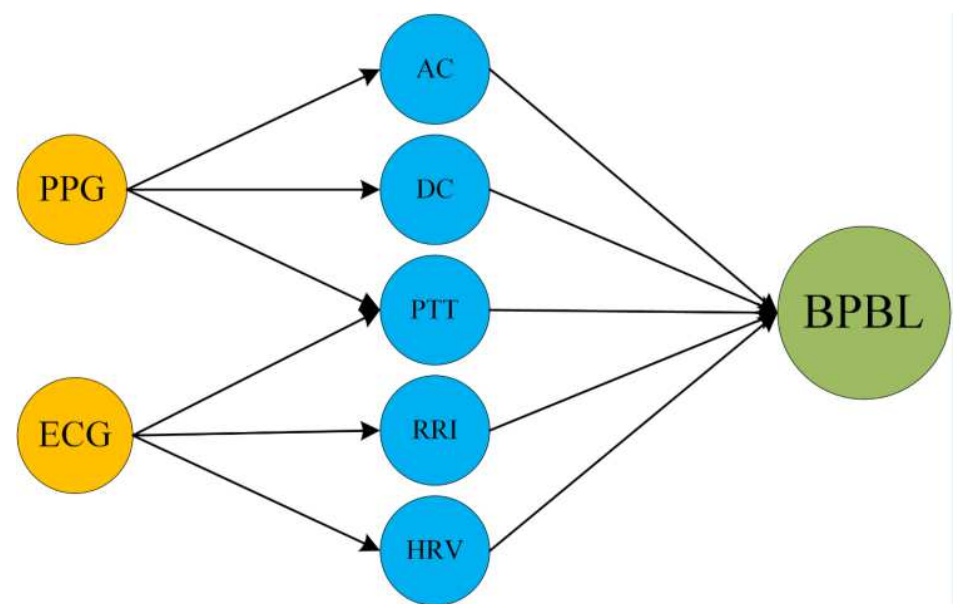
calculated by ECG and PPG. These characteristic parameters are used to estimate the BPBL model. 

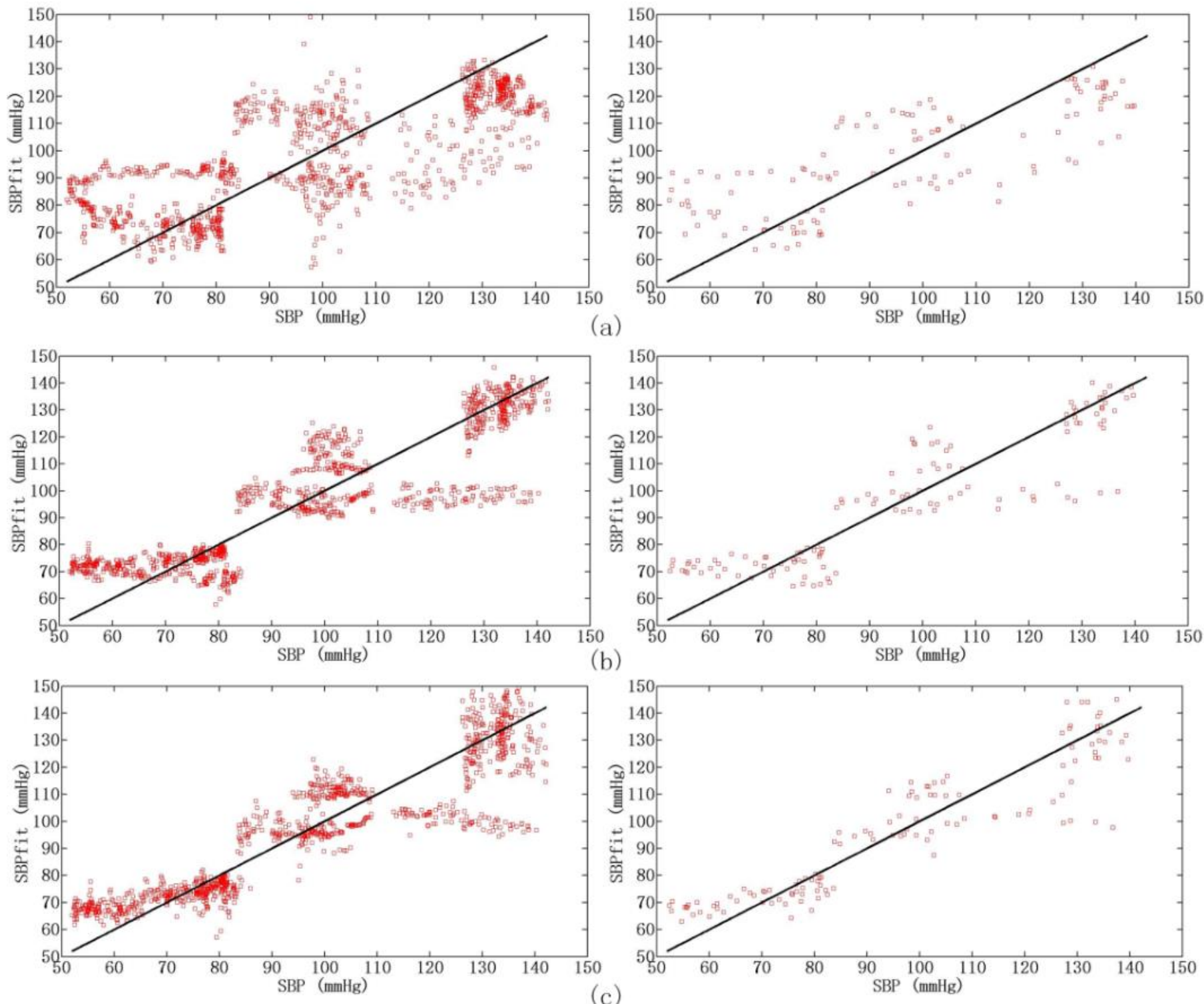

Fig. 6. The results of the three blood loss models. The left side of the picture is the training set, and the right side is the prediction set. (a) The experimental results of PTT model. (b) The experimental results of RRI model. (c) The experimental results of HRV model.

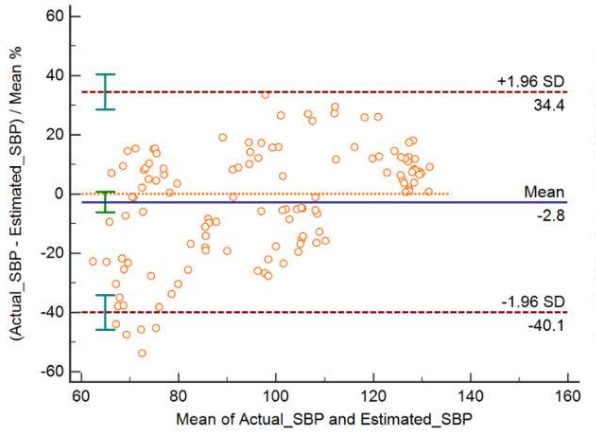

(a)

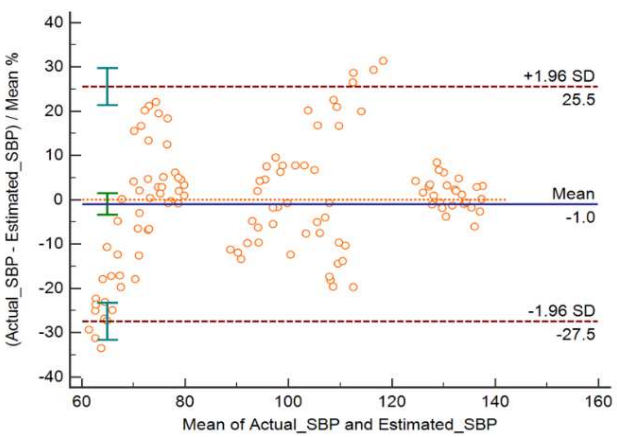

(b)

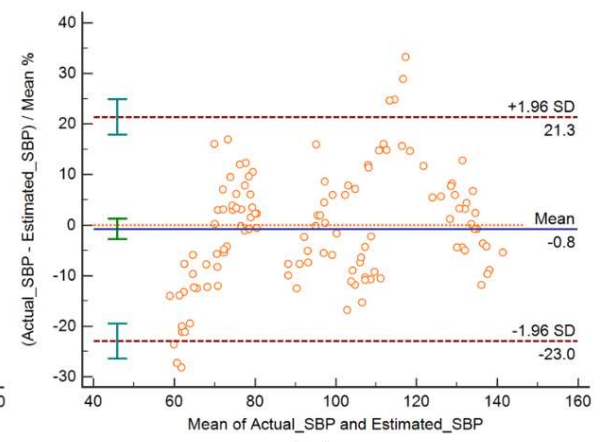

(c)

Fig. 7. Bland-Altman plot of the difference between actual SBP and estimated SBP in three models. (a)the difference of PTT model. (b) the difference 
Table 1. Comparison of input parameters of three models.

\begin{tabular}{cc}
\hline \hline BPBL Model & Input Parameters \\
\hline PTT model & PTT, AC, DC, AC/DC \\
RRI model & PTT, AC, DC, AC/DC, RRI, PTT/RRI \\
HRV model & PTT, AC, DC, AC/DC, SDNN, RMSSD, HF \\
\hline \hline
\end{tabular}

The input parameters of the PTT model are simply extracted by combining the ECG and PPG signals, and the input parameters of the RRI model and the HRV model are added with RRI and HRV based on PTT model, respectively.

Table 2. Comparison of experimental results of three models.

\begin{tabular}{ccccccc}
\hline \hline Blood Loss Model & $\begin{array}{c}\text { Optimal } \\
\text { Factor }\end{array}$ & Rt & RMSEC & Rp & RMSEP & P-value \\
\hline PTT model & 3 & 0.73 & $18.1 \mathrm{mmHg}$ & 0.77 & $16.8 \mathrm{mmHg}$ & $* * *$ \\
RRI model & 5 & 0.89 & $12.2 \mathrm{mmHg}$ & 0.89 & $11.9 \mathrm{mmHg}$ & $* * *$ \\
HRV model & 6 & 0.91 & $11.1 \mathrm{mmHg}$ & 0.92 & $10.6 \mathrm{mmHg}$ & $* * *$ \\
\hline \hline
\end{tabular}




\section{Figures}

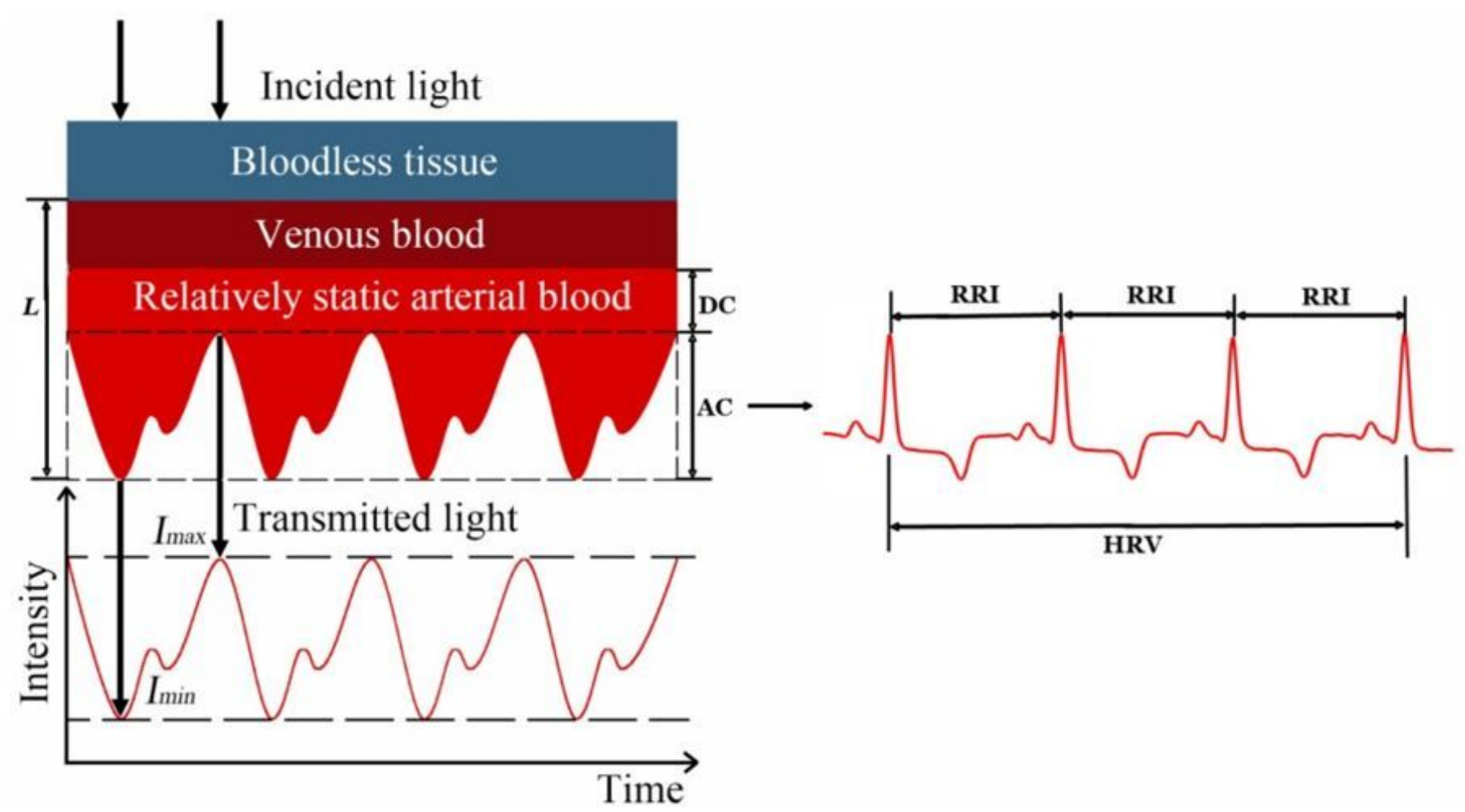

Figure 1

Basic theory of blood loss. On the left side of the picture, the blood in the blood vessel is composed of dynamic components and static components. The near-infrared light emitted by PPG passes through the bloodless tissue layer and blood of the human body and reflects back and is received by the sensor. Since the light intensity change curve of the reflected light is closely related to the pulse, PPG technology can be used to obtain the pulse wave waveform, and the AC and DC can be extracted from the pulse wave. In addition, AC is depending on the heart rate, so we calculated RRI to reflect blood changes together. 


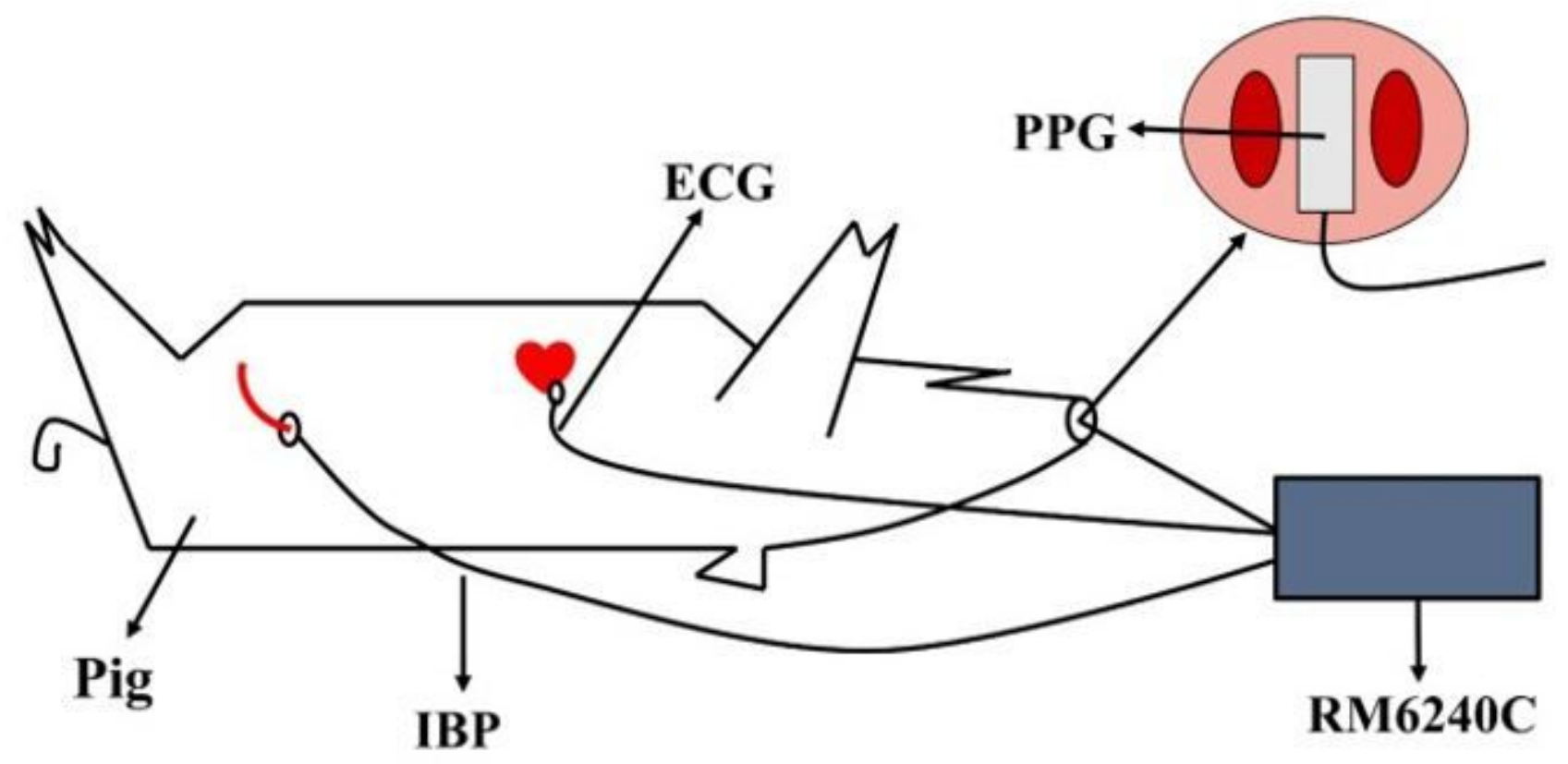

Figure 2

Experimental scene diagram. The picture shows the side view of the pig on the operating table. During the experiment, we used the Chengdu Instrument RM6240C multi-physiological channel acquisition system to collect signals. We collected the PPG, ECG and IBP signals of the pig at the nose, chest and left femoral artery, respectively.

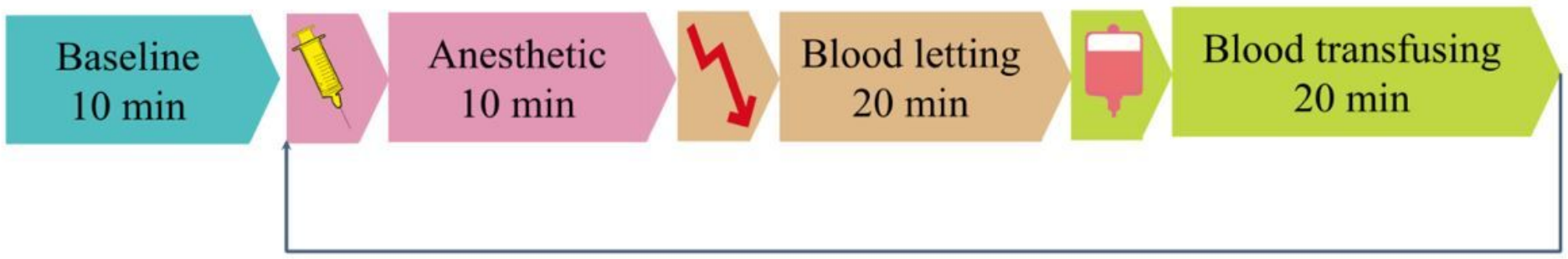

Figure 3

Introduction to the experimental process. During the experiment, bloodletting and blood transfusion were performed when the physiological state of the pig was stable, and the specific number of times was determined by the physiological state of the pig. See text for further details. 


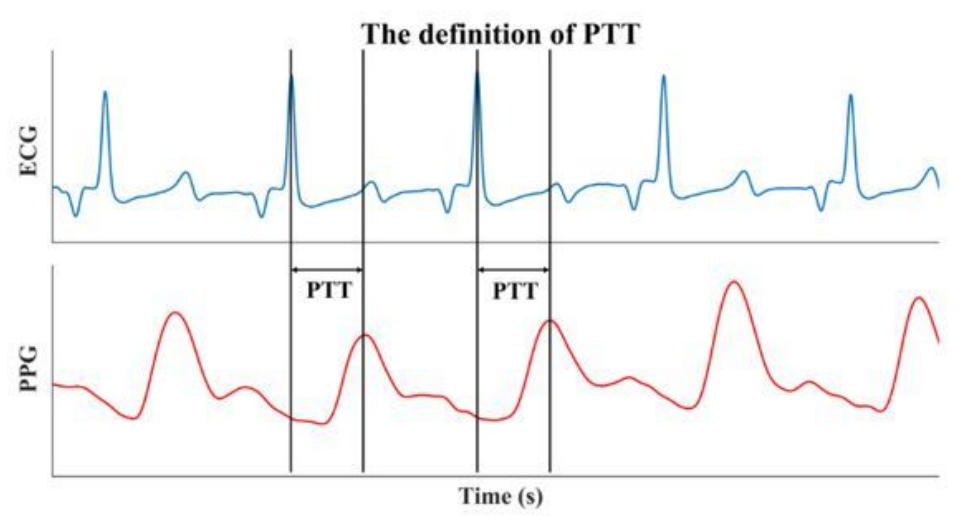

(a)

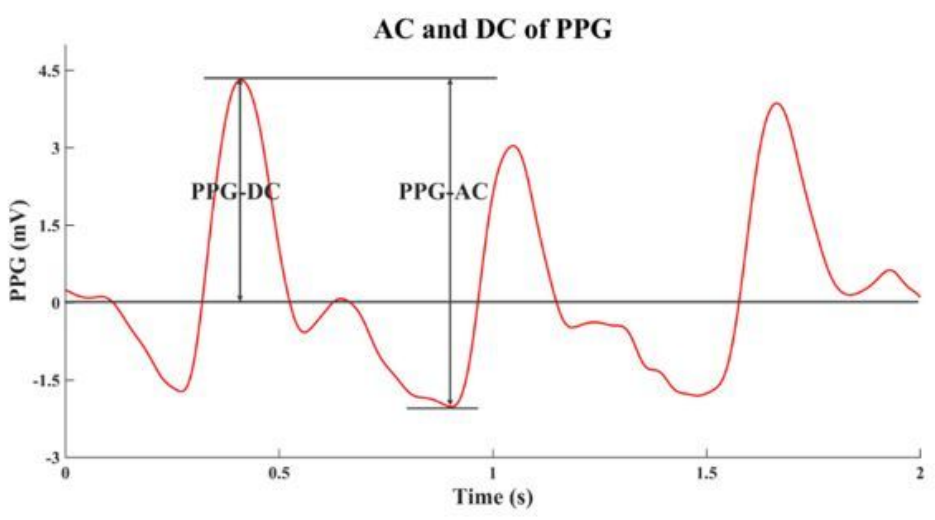

(b)

\section{Figure 4}

The extraction of PTT, AC, and DC. (a) The PTT refers to the time that the pulse wave travels from the heart to the surrounding branch blood vessels during the pumping of the heart. (b) DC and AC refer to the peak value and amplitude of PPG respectively.

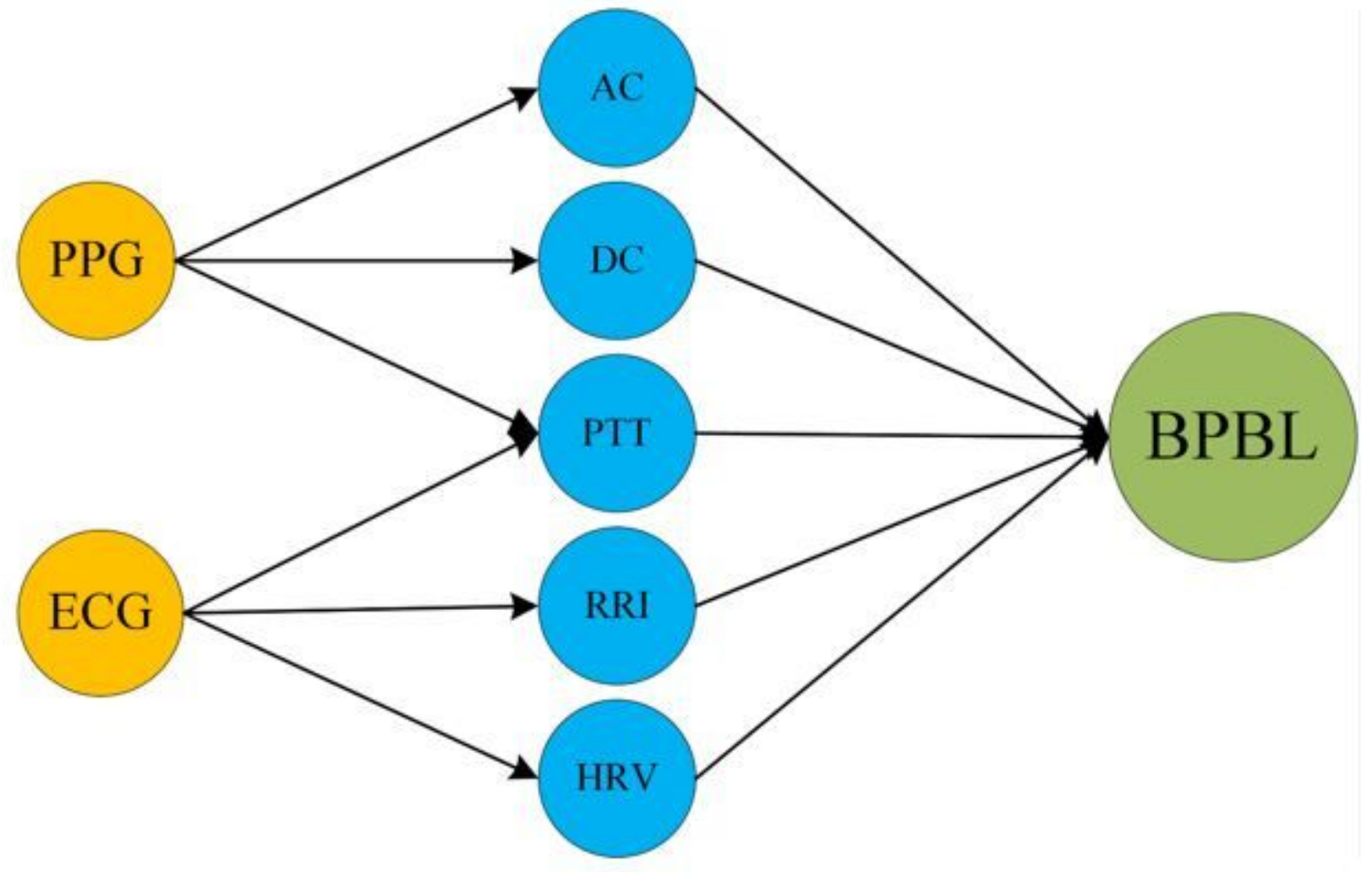

Figure 5

Parameters for estimating BPBL. AC and DC are extracted from PPG, RRI and HRV are extracted from ECG, and PTT is calculated by ECG and PPG. These characteristic parameters are used to estimate the BPBL model. 

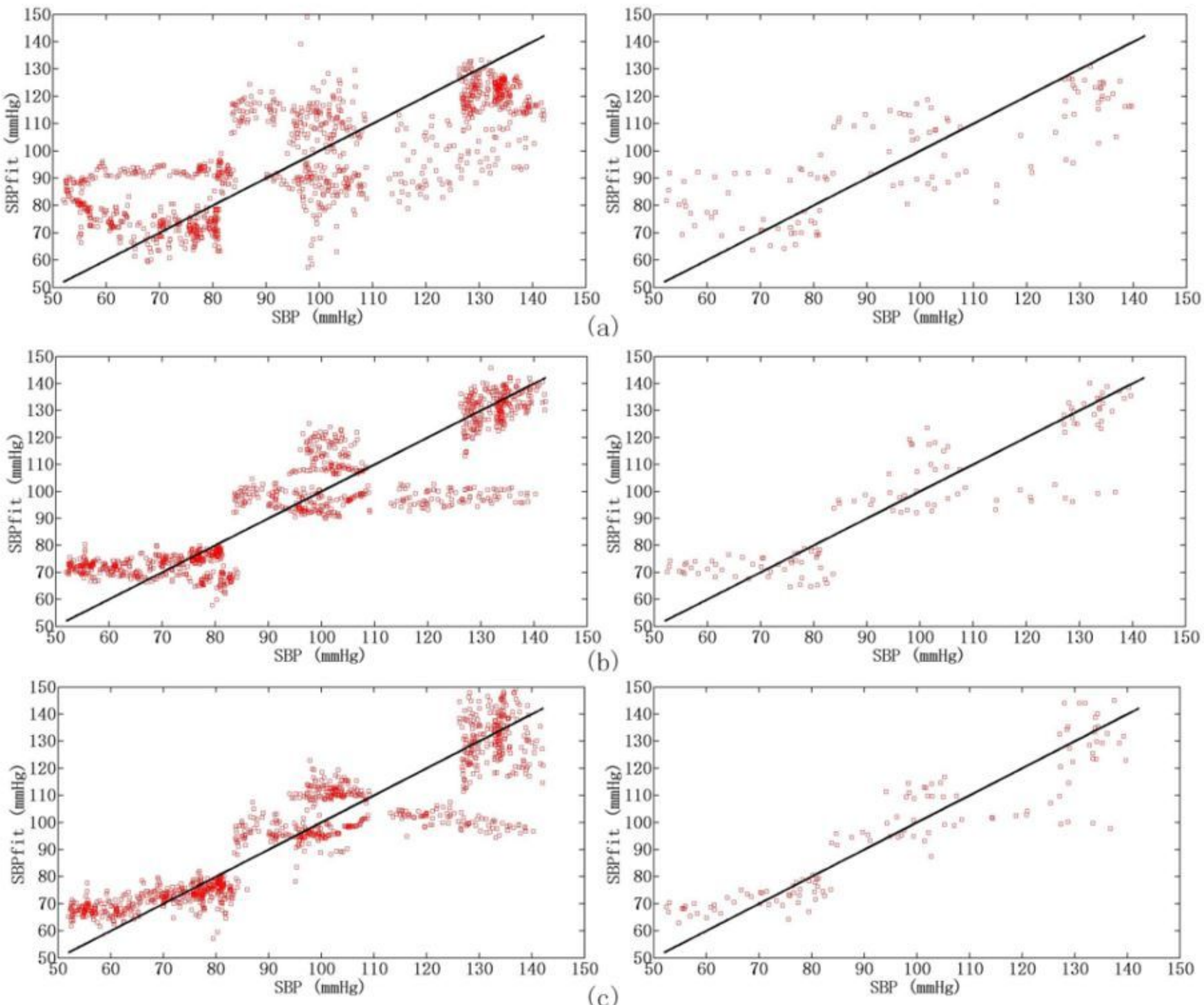

Figure 6

The results of the three blood loss models. The left side of the picture is the training set, and the right side is the prediction set. (a) The experimental results of PTT model. (b) The experimental results of RRI model. (c) The experimental results of HRV model.

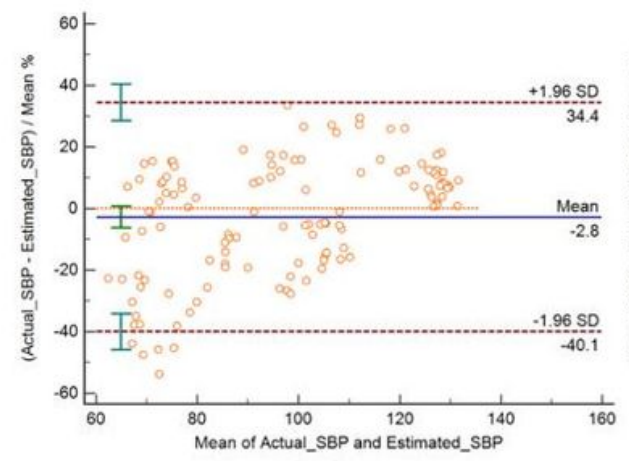

(a)

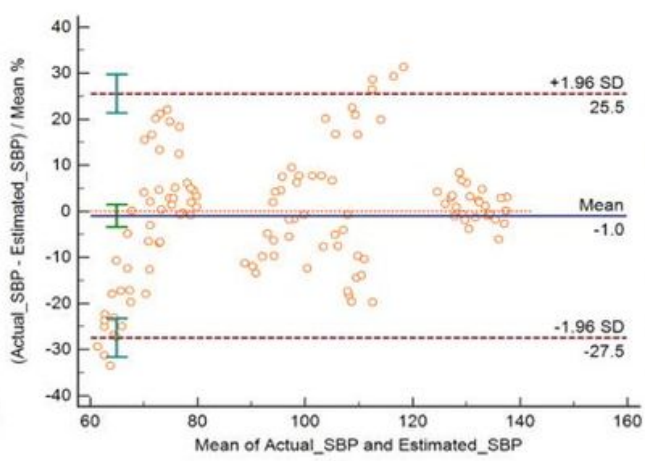

(b)

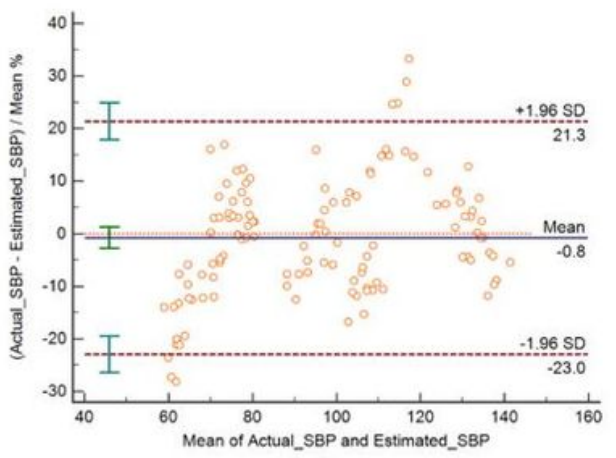

(c) 
Figure 7

Bland-Altman plot of the difference between actual SBP and estimated SBP in three models. (a)the difference of PTT model. (b) the difference of RRI model. (a)the difference of HRV model. 